\title{
Choroidal Thickness and its Correlations with Ocular Parameters in Primary Open-angle Glaucoma
}

\author{
Dunyamin Kutluksaman, 1, * (1) Guliz Fatma Yavas,, , * (1) Sibel Inan,, * (1) Mustafa Dogan,, * \\ Umit Ubeyt Inan ${ }^{4, *}$ \\ ${ }^{1}$ Department Ophthalmology, Necip Fazil City Hospital, Kahramanmaras, Turkey \\ ${ }^{2}$ Department of Ophthalmology, Hacettepe University, Ankara, Turkey \\ ${ }^{3}$ Department of Ophthalmology, Afyonkarahisar Health Sciences University, Afyonkarahisar, Turkey \\ ${ }^{4}$ Department Ophthalmology, Parkhayat Hospital, Afyonkarahisar, Turkey \\ ${ }^{*}$ Former affiliation: Department of Ophthalmology, Afyon Kocatepe University, Afyonkarahisar, Turkey
}

\begin{abstract}
Objectives: To evaluate the relationships between macular choroidal thickness (mCT) and ocular parameters, such as optic nerve head $(\mathrm{ONH})$ and multifocal electroretinogram (mf-ERG) parameters, in cases with primary open-angle glaucoma (POAG).

Methods: This controlled and prospective clinical trial included 49 patients with POAG diagnosed for the first time and 47 healthy participants. Macular CTs, ONH and mf-ERG parameters were measured, and the examination findings were recorded at baseline and follow-ups.

Results: In the POAG group, the mean $\mathrm{mCT}$ was $254.92 \pm 37.65 \mu \mathrm{m}$ at baseline, and it was $235.6 \pm 38.48 \mu \mathrm{m}$ at 3-month and was $237.55 \pm 37.27 \mu \mathrm{m}$ at 6-month. In the glaucoma group, there was a significant decrease in the first three months despite the treatment, but no significant change was observed in the next three months. In the healthy group, the mean mCTs were $287.78 \pm 26.77 \mu \mathrm{m}, 285.48 \pm 25.58 \mu \mathrm{m}$ and $285.02 \pm 27.44 \mu \mathrm{m}$ at baseline, at 3-month and at 6-month, respectively. No significant change was observed in the control group throughout the process. However, the mean mCT values in the glaucoma group were significantly thinner in all controls compared to the healthy group $(p<0.05)$. Furthermore, significant correlations were found between $C T$ and some $\mathrm{ONH}$, as well as mf-ERG parameters.

Conclusion: The choroid can play an important role in the pathogenesis of glaucoma. Significant correlations in parameters support this relationship. We have observed that the glaucomatous effect initiated first in the inferior quadrant of ONH.

Keywords: Enhanced depth imaging optical coherence tomography, macular choroidal thickness, multifocal electroretinogram, optic nerve head, primary open-angle glaucoma.
\end{abstract}

\section{Introduction}

Glaucoma is a progressive and chronic optic neuropathy resulting in loss of visual field $(I, 2)$. Although the cause of pathological processes resulting in degeneration of the retinal ganglion cells is thought to be highly variable, the most common cause and pathophysiology is the increase in intraocular pressure $(3,4)$. Although many pathophysiological processes have been proposed in the formation of glaucomatous optic neuropathy (GON), one of the most discussed processes in recent years is that the radial peripapillary cap- illary network plays an important role in the pathogenesis of glaucoma (5-7). In recent years, optical coherence tomography (OCT) angiography showed that the vascular network was significantly associated with a decrease in retinal nerve fiber layers (RNFL) thickness $(8,9)$. The choroid and retinal circulatory system collaterals play an important role in the vascular supply of the prelaminar nerve fiber layer and the inner retinal layers at the optic nerve head $(\mathrm{ONH})$, which has a highly complex vascular supply (10). Choroidal vascular supply may play a role in the pathogenesis of glaucoma is supported by many studies; however, there are many oppos- 
ing studies about which type of thickness (peripapillary or macular choroidal thickness) contribute more (II-I5).

Alongside developments and new technologies, generally accepted and most commonly used methods for diagnosis and follow-up of glaucoma are a computerized visual field (perimetry) and the OCT, which is capable of performing a detailed analysis of the $\mathrm{ONH}$ concerning GON. While perimetry can provide important information for the regional damage of the RNFL, ONH imaging using OCT (ONH-OCT) can provide quantitative information about total damage at $\mathrm{ONH}$, where RNFLs are collected, concerning GON. However, perimetry and OCT are the devices that can only give us results after damage occurs in RNFLs. Well then, is it possible to monitor the pathophysiology of GON in earlier stages? One of the main issues we need to know for this question is whether or not the outer retinal layers play a role in the optic neuropathy formation mechanism. As previously mentioned, if the choroidal radial peripapillary vascular network has an important role in GON, external retinal cells, which have the vascular supply from the macular choroid associated with the peripapillary choroid, can be expected to be affected. The method that can help us in this matter is multifocal electroretinogram (mfERG), which can give us localized comments, especially about photoreceptor cells in the outer retinal layers. As is known, electroretinography (ERG) is an electrical mass response that shows the sum of the electrical activity obtained from retinal cells (16). mf-ERG techniques were developed to perform the topographic measurement of retinal electrophysiological activity in different retinal areas (17). Although it is generally accepted that mf-ERG provides more information about photoreceptor cells and/or bipolar cells, there are some studies supporting that mf-ERG may also provide information about retinal ganglion cells in cases with glaucoma $(17,18)$. Therefore, it is important to investigate the role of mf-ERG in glaucoma concerning evaluating the activity of external retinal cells, which has vascular supply via choroid, as well as ganglion cells.

The primary objectives of this study were to evaluate macular choroidal thickness ( $\mathrm{m}-\mathrm{CT})$ in newly diagnosed early-stage open-angle glaucoma and its correlation with $\mathrm{ONH}$ OCT and mf-ERG parameters.

\section{Methods}

This prospective controlled clinical trial was performed in accordance with the principles of the Declaration of Helsinki. For this study, ethical approval was obtained from the clinical trials ethics committee of the University of Afyon Kocatepe - 20I4/04-74), and the study protocol is registered on www. ClinicalTrials.gov (NCT03966560). A total of 102 participants, 5 I patients of which were diagnosed with POAG for the first time and who were initiated antiglaucoma medication, as well as age-matched $5 \mathrm{I}$ healthy volunteers, were included in this study that took place between January 2014 and April 2015 at
Afyon Kocatepe University of Department of Ophthalmology. Firstly, the procedures to be done were explained to the participant candidates who met the criteria for this trial and an information form was given to them. Participants who wanted to volunteer were included in this study after obtaining consent documents.

\section{Diagnosis of the Primary Open-Angle Glaucoma}

POAG was diagnosed by these criteria: the measurement of intraocular pressure with Goldmann applanation tonometry (AT900 Haag-Streit Diagnostics, Haag-Streit AG) being above $2 \mathrm{I} \mathrm{mmHg}$, the detection of an open angle by gonioscopy (Volk G-4, Volk Optical Inc), the detection of glaucomatous optic disc pitting by 90 diopter lens (SuperField NC, Volk Optical Inc) in slit-lamp fundus eye examination, OCT imaging (Cirrus HD 4000, Carl Zeiss Meditec AG) of ONH and characteristic visual field defect detection in SITA 24: 2 Humphrey visual field test (Carl Zeiss Meditec AG).

Participants over the age of 40 years who were diagnosed with POAG for the first time and had the best-corrected visual acuity (BCVA) of 0.6 and above were included in this study for the POAG group. Participants with secondary cause of glaucoma, angle closure in gonioscopic examination (Grade 0, I, and 2 according to Shaffer's classification system), corneal opacity or cataract at the level that may affect imaging, vitreous pathology, intravitreal hemorrhage that may affect fundus appearance, retinal pathology, chorioretinopathy, optic neuropathy, optic disc pathology, spherical refractive error of $6 \mathrm{D}$ and above, cylindrical refraction error of 3D and above and systemic diseases which may affect ocular blood flow, were excluded. Participants were also excluded from this study if these situations developed in the volunteers during the study.

All patients underwent a routine ophthalmologic examination before being included in this study. Visual acuity values were recorded. After biomicroscopic examination, IOP was measured using applanation tonometry. Measurement of central corneal thickness (CCT) was performed with topical anesthesia (proparacaine hydrochloride ophthalmic solution 5\%) using the contact ultrasound pachymeter (Pocket II, Quantel Medical Inc). 360-degree gonioscopic angle examination was performed with four mirror lenses. The gonioscopic angle examination was recorded considering the Schaffer classification system.

\section{OCT Parameters and Choroidal Thickness}

ONH OCT parameters, such as neuroretinal rim area (RIM), disc area (DISCA), average cup-to-disc ratio (ACDR), vertical cup-to-disc ratio (VCDR), cup volume (CV) and RNFL thicknesses of the ODH quadrants, as well as CTs, were recorded using Cirrus HD 400 OCT. CTs were measured at the fovea, the $3 \mathrm{~mm}$ nasal of the fovea and the $3 \mathrm{~mm}$ temporal of the fovea using enhanced depth imaging OCT (EDI-OCT) mode of the same device. 


\section{Multifocal Electroretinogram (mf-ERG)}

All mfERG tests were performed by the same technician with the same device (Metrovision Monpack 3, Metrovision, France). Before the test, maximum pupil dilation was achieved with $1.0 \%$ tropicamide administration. The refractive error was corrected according to $33 \mathrm{cms}$. ERG-jet electrode was used as the active electrode during the test. ERGjet electrode was placed on the cornea after the application of a drop of $0.5 \%$ proparacaine $\mathrm{HCl}$. Before the ground electrode and the reference electrode were placed, the surface skin and oily layer skin, which were known to have low electrical conductivity, were cleaned. Subsequently, the ground electrode was placed in the middle of the forehead, and the reference electrode was placed in the temporal region $\mathrm{I} \mathrm{cm}$ ahead of the external canthus.

The electrodes were connected using the junction box, and the signals were processed. The other eye was closed and an infrared camera monitored the fixation during the study. MERG6IB test was applied according to the International Society for Clinical Electrophysiology of Vision criteria. Image patterns consisting of $6 \mathrm{I}$ hexagons set to create equal signal were used on the monitor screen and the recordings from the $6 \mathrm{I}$ regions of the retina were recorded in approximately five minutes. The screen resolution was set to $1024 \times 768$ pixels. The participant was placed $33 \mathrm{~cm}$ away from the screen during the test, and a horizontal \pm 30 -degree area and a vertical \pm 24 -degree area were stimulated. The stimulus frequency was $17 \mathrm{~Hz}$ and the luminance was $100 \mathrm{~cd} /$ $\mathrm{m}^{2}$. The background light was set to $30 \mathrm{~cd} / \mathrm{m}^{2}$. When there was no stimulus during the test, the existing electrical activity and noise level were recorded; however, the results with noise levels above $5 \mu \mathrm{V}$ were not evaluated. If the number of fixation loss and rejected stimuli exceeded $20 \%$ of total stimuli, the results of the test were not included in this study. For the wave of "First row-Kernel" in each ring, the amplitude and impulse time of the $\mathrm{NI}$ wave and the amplitude and impulse time of the PI wave were calculated in concentric ring analysis. In the concentric ring analysis, according to the fixation, the Ist ring represented the 0-5 degree retinal area, the second ring represented the areas of 5-10 degrees, the third ring represented the areas of $10-15$ degrees, and the $4^{\text {th }}$ ring represented the area of 15 degrees as well as it is a periphery. Mean amplitude (nanovolt) and time of impulse (milliseconds) were recorded for the whole ring analysis.

\section{Statistical Analysis}

A total of 18.240 data from 96 cases were coded and transferred to the computer program. SPSS 20.0 (SPSS Inc) software was used for statistical evaluation. Firstly, the normal distribution of the values was tested by testing the normality. For the test of normality, the Shapiro-Wilk test was used for cases where the number of participants was higher than 50 and the Kolmogorov-Smirnov test was used when the number of participants was less than 50. In the comparison of a single parameter between the two groups, independent-samples t-test was used for the groups with normal distribution and the Mann-Whitney $U$ test was used for the groups that did not conform to the normal distribution. For evaluating the changes during the process of repeated measurements, repeated measures analysis of variance (ANOVA) was used for groups with normal distribution and the Friedman test was used for groups' cases that did not comply with normal distribution. $P$ results that were $\leq 0.05$ were deemed statistically significant. Correlations between the parameters were analyzed by Pearson bivariation correlation analysis for groups with normal distribution, and Spearman bivariation correlation analysis was used in cases where one of the groups did not comply with normal distribution. In correlation analysis, + values were correlated in the same direction, - values were considered as inverse correlation. In addition, a correlation was deemed to be present in cases the correlation coefficient values $(r)$ were $\geq \pm 0.3$ and a significance level of $p$-values that were $\leq 0.05$ were accepted as significant correlation.

\section{Results}

Two participants in the glaucoma group and four participants in the healthy group were excluded from this study because of failure to comply with follow-up examinations and mfERG tests. Table I shows the baseline values of enrolled patients included in this study.

Table I. Baseline values of enrolled patients

\begin{tabular}{lcc} 
& Glaucoma Group & Healthy Group \\
\hline No. (\%) & $49(51 \%)^{*}$ & $47(49 \%)^{*}$ \\
Age, (SD), [range], years & $58.22(11.06)[40-82]$ & $58.23(10.18)[42-79]$ \\
Sex, Male (\%) & $23(47 \%)^{* *}$ & $21(45 \%)^{* *}$ \\
Side, Right (\%) & $25(51 \%)^{* *}$ & $23(49 \%)^{* *}$ \\
CCT, (SD), [range], $\mu \mathrm{m}$ & $549.13(14.39)[515-598]$ & $546.72(10.63)[522-575]$ \\
\hline
\end{tabular}


In the POAG and the healthy group, the BCVA levels of the initial and follow-up periods are shown in Table 2. While repeated measures ANOVA showed that there was a significant decrease in the mean BCVA in the POAG group during the 6-month follow-up; however, a significant change was not observed in the healthy group during the study (Table 2). The mean IOPs during the study for the POAG and the healthy groups are shown in Table 2. The mean IOPs of the group showed a significant decrease in the first I-month after the treatment; however, no significant change was observed for the next five months. There was no significant change in the mean IOPs in the healthy group for six months (Table 2).

\section{OCT Parameters}

Table 3 shows the mean values of OCT parameters for the POAG and the healthy groups. There was no significant change in within-group evaluations for average RNFL thickness, RIM, ACDR, VCDR, and cup volume values, but there were significant differences between groups. There were no significant changes in the mean DISCA values within the groups and between the groups for 6-months ( $p>0.05$ for each follow-up). The mean disc areas $\left(\mathrm{mm}^{2}\right)$ in the POAG group $(n=49)$ were $2(0.44)$ at baseline, 2.05 (0.48) at I-month, $2.1(0.5)$ at 3-month and $2.02(0.45)$ at 6-month. The mean disc areas $\left(\mathrm{mm}^{2}\right)$ in the healthy group $(n=47)$ were 2.02 (0.38), 2.05 (0.37), $2(0.5)$ and $2(0.39)$ respectively. Friedman test showed no significant changes in the mean disc areas from baseline to 6-month in the both groups $(p>0.05)$.

\section{Retinal Nerve Fiber Layers Thicknesses of Optic Disc Quadrants}

The mean RNFL thicknesses according to the optical disc quadrants are shown in the Table 3. When the mean RNFL thicknesses were compared between the groups, the superior, temporal and nasal RNFL thicknesses did not change during the process, but there were significant differences between the two groups. However, the mean RNFL thicknesses of the inferior quadrant significantly decreased only in the POAG group.

\section{Multifocal Electroretinogram Parameters}

\section{The First Ring (RI)}

For the first ring, mf-ERG parameters were compared between both groups: there were significant differences between groups in only the mean amplitudes of $\mathrm{NI}$ wave $(\mathrm{NIaRI})$ at baseline and 6-month $(p<0.05)$. The mean amplitudes of NI wave $\left(\mathrm{nv} / \mathrm{deg}^{2}\right)$ in the POAG group $(\mathrm{n}=49)$ were -34.84 (II.2) at baseline and -35.23 (12.9) at 6-month. The mean amplitudes of $\mathrm{NI}$ wave $\left(\mathrm{nv} / \mathrm{deg}^{2}\right)$ in the healthy group $(n=47)$ were $-30.39(9.5)$ and $-27.77(12.5)$ respectively. A significant change from baseline to 6-month was observed only in the mean amplitude of the N2 wave, and it was seen only in the POAG group (Table 4). No significant changes were observed for both groups when the changes in intragroup values were evaluated in other parameters for six months $(p>0.05)$.

\section{The Second Ring (R2)}

Comparison of second ring parameters in the POAG and the healthy groups: the mean values of $\mathrm{NlaR2}$ (the amplitude of NI wave - Ring 2) between the groups were found to be significantly lower in the POAG group at baseline and no significant differences were observed in subsequent periods. The mean amplitude of $\mathrm{NI}$ wave $\left(\mathrm{nv} / \mathrm{deg}^{2}\right)$ in the POAG group $(n=49)$ was $-25.06(6.62)$ at baseline and was -22.15 $(6.91)$ in the healthy group $(n=47)$ at baseline $(p<0.05)$. Significant differences between the groups in other parameters

Table 2. The mean best-corrected visual acuities and intraocular pressures during the study

\begin{tabular}{|c|c|c|c|}
\hline Visual Acuity (SD) [range] decimal & Glaucoma group, $n=49$ & Healthy group, $n=47$ & p-value* \\
\hline I-Month & $0.86(0.12)[0.7-1.0]$ & $0.92(0.09)[0.8-1.0]$ & 0.015 \\
\hline 3-Month & $0.86(0.12)[0.6-1.0]$ & $0.92(0.09)[0.8-1.0]$ & 0.017 \\
\hline 6-Month & $0.84(0.13)[0.6-1.0]^{* *}$ & $0.91(0.09)[0.8-1.0]^{* * *}$ & 0.007 \\
\hline I-Month & I5.63 (2.23) [12-20] & $16.57(1.67)[13-20]$ & 0.039 \\
\hline 3-Month & $15.46(1.9)[11-19]$ & $15.86(1.77)[13-20]$ & 0.43 \\
\hline 6-Month & $15.12(1.69)[12-18]^{* *}$ & $15.85(1.56)[|3-2|]^{* * *}$ & 0.08 \\
\hline
\end{tabular}

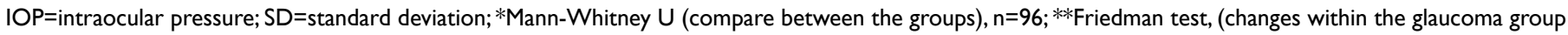
from baseline to 6-month), $\mathrm{p}<0.05, \mathrm{n}=49$; ***Friedman test, (changes within the halthy group from baseline to 6 -month), $\mathrm{p}>0.05, \mathrm{n}=47$. 
Table 3. The mean optic nerve head OCT parameters in both groups during the study

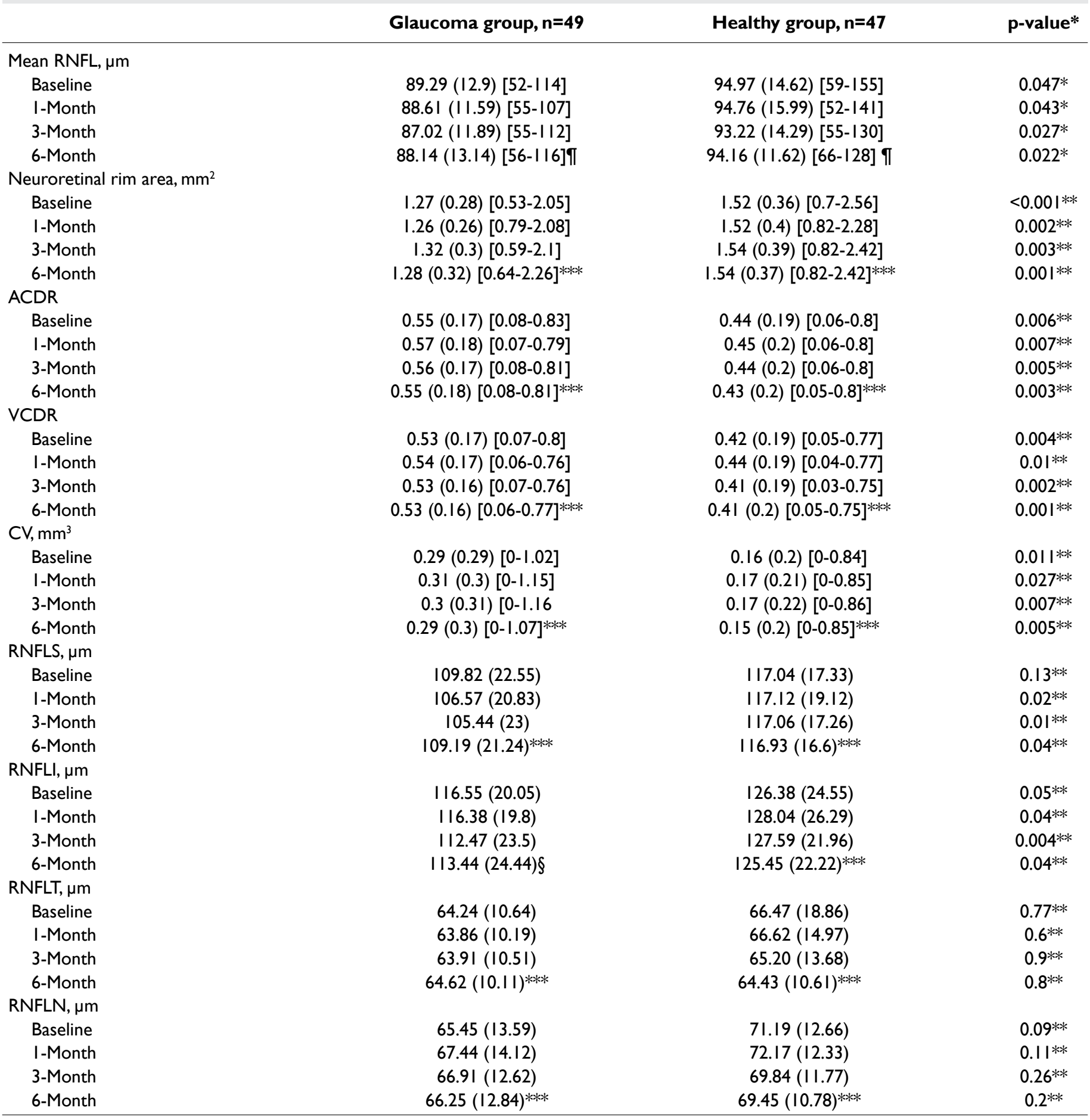

$A C D R=$ average cup to disc ratio; $C V=$ cup volume; $R N F L=$ retinal nerve fiber layer thickness; $R N F L S=$ superior; $R N F L I=$ inferior; $R N F L T=$ temporal; $R N F L N=$ nasal; $\mathrm{VCDR}=$ vertical cup to disc ratio; *Student's t-test (Compare between the groups); **Mann-Whitney U Test (Compare between the groups); ***Friedman test, (Changes within the group from baseline to 6-month), $p>0.05$; §Friedman test, (Changes within the group from baseline to 6-month), $p<0.05 ; \uparrow T h e$ repeated measures ANOVA, (Changes within the group from baseline to 6-month), $p>0.05$.

were not observed. There were no significant changes from baseline for each group during the study.

\section{The Third Ring (R3)}

When both groups were evaluated concerning third ring parameters, although there were no significant differences concerning the mean values of $\mathrm{NIaR} 3$ in the POAG and the healthy groups, only the mean $\mathrm{NlaR} 3$ values in the parameters decreased significantly only in the POAG group during 
Table 4. The changes in the mean amplitudes of $\mathrm{NI}$ waves in ring-3 and N2 waves in ring-I during the study

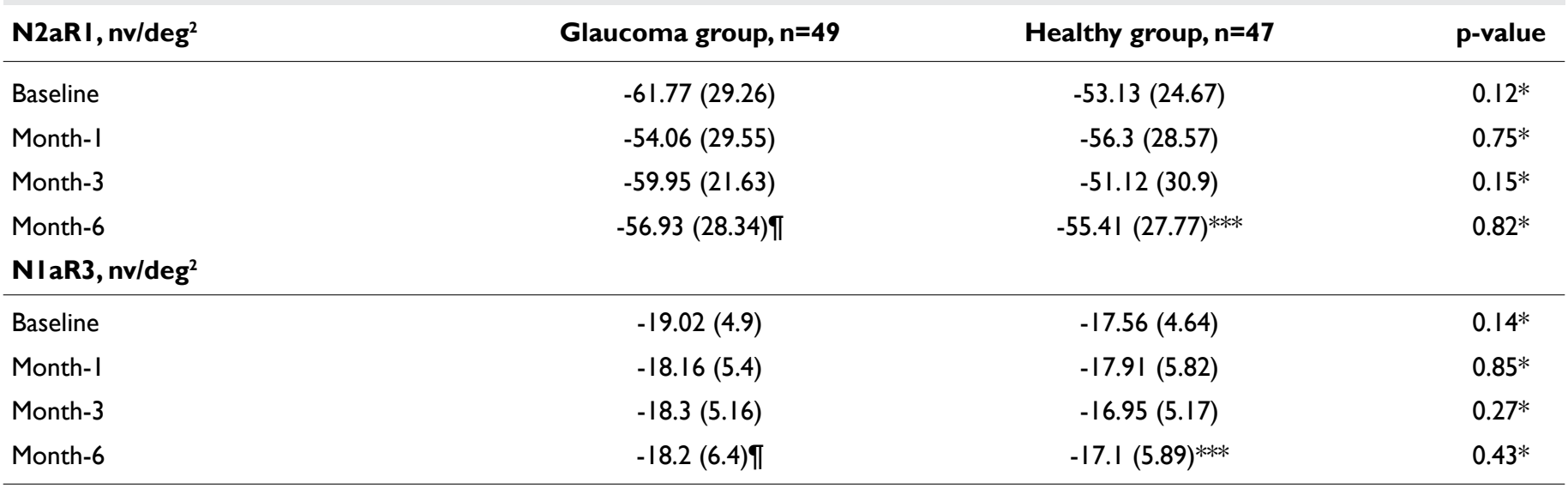

N2aRI: N2 wave amplitudes in ring-I, NIaR3: NI wave amplitudes in ring-3; *Student's t-test (Compare between the groups); ${ }^{* * *}$ The repeated measures ANOVA, (Changes within the group from baseline to 6-month), $\mathrm{p}>0.05$; $\uparrow$ The repeated measures ANOVA, (Changes within the group from baseline to 6-month), $\mathrm{p}<0.05$.

this study (Table 4). Other parameters in R3 did not differ between the two groups, and no significant changes were observed for each group.

\section{The Fourth Ring (R4)}

No significant differences were found between the groups in R4 analysis.

\section{Choroidal Thicknesses (CTs)}

When the POAG group and the healthy group were compared concerning the mean CTs, the mean CTs of the POAG group were significantly thinner than the healthy group in all of the follow-ups (Table 5). In addition, even though there was a significant decrease in the POAG group, no significant change was observed in the healthy group during the study (Fig. I).

\section{Correlations}

\section{Correlations between mf-ERG Parameters and CT}

When the mean $\mathrm{NI}$ wave amplitude values for each ring were analyzed for correlation with the mean $\mathrm{CT}$ s of each control period: while there were no correlations between $\mathrm{NI}$ amplitudes and CTs in the POAG group, significant positive correlations between CTs, and the mean amplitudes of

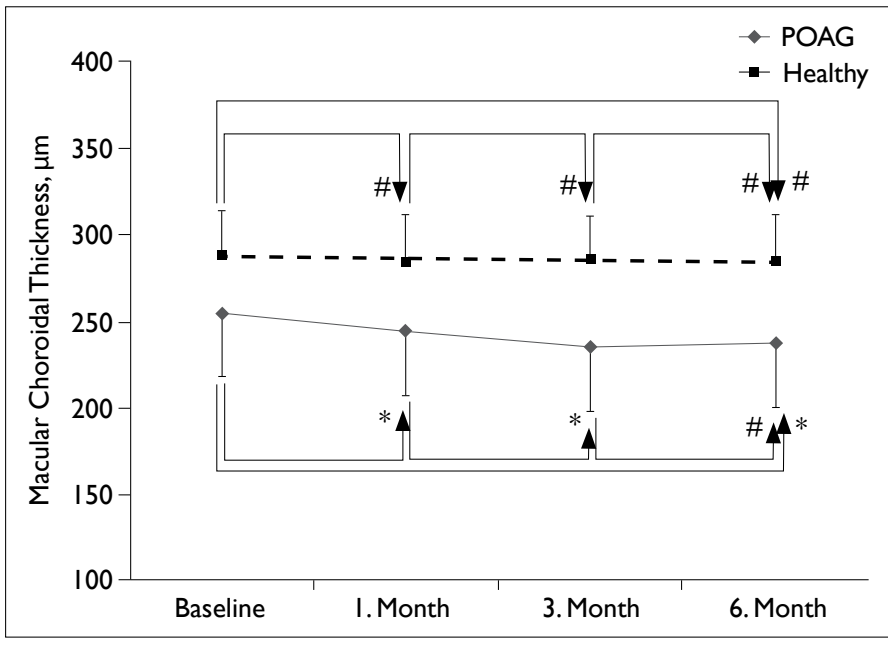

Figure I. The mean macular choroidal thicknesses in the primary open-angle glaucoma and the healthy groups during the study. POAG=primary open-angle glaucoma; Repeated measures ANOVA test showed significant decreases only in the POAG group from baseline to 6-month. $\left(\# p>0.05,{ }^{*}<0.05\right)$.

$\mathrm{NI}$ wave at 6-month in R2, R3, and R4 were detected in the healthy group (Table 6).

Table 5. The mean choroidal thicknesses of groups during the study

\begin{tabular}{lccc} 
Choroidal Thickness, (SD), [range], $\boldsymbol{\mu m}$ & Glaucoma group, $\mathbf{n = 4 9}$ & Healthy group, $\mathbf{n = 4 7}$ & $\mathbf{p - v a l u e *}$ \\
\hline Baseline & $254.92(37.65)[189-370]$ & $287.78(26.77)[222-334]$ & $284.14(28.09)[219-338]$ \\
Month-I & $243.78(36.47)[158-32 I]$ & $285.48(25.58)[219-340]$ & $<0.01$ \\
Month-3 & $235.6(38.48)[141-316]$ & $285.02(27.44)[217-344]^{* * *}$ & $<0.01$
\end{tabular}

*Student's t-test showed significant differences between groups during the study, $n=96$; ** The repeated measures ANOVA showed significant decrease in the glaucoma group during the study, $\mathrm{p}<0.01, \mathrm{n}=49 ; * * *$ The repeated measures ANOVA showed no significant change in the healthy group during the study, $\mathrm{p}=0.23, \mathrm{n}=47$. 
Table 6. The correlations between macular choroidal thicknesses and the amplitudes of $\mathrm{NI}$ and N2 waves in Ring 2-3-4 in the healthy group

\begin{tabular}{|c|c|c|c|c|}
\hline & CT baseline & CT I- month & CT 3- month & CT 6- month \\
\hline \multicolumn{5}{|l|}{ Nla-Ring 2} \\
\hline Pearson corr. & $0.43 *$ & 0.26 & $0.47^{*}$ & $0.48 *$ \\
\hline $\mathrm{p}$-value & 0.005 & 0.12 & 0.003 & 0.003 \\
\hline \multicolumn{5}{|l|}{ Nla-Ring 3} \\
\hline Pearson corr. & $0.4^{*}$ & 0.29 & $0.38 *$ & $0.4^{*}$ \\
\hline $\mathrm{p}$-value & 0.01 & 0.08 & 0.017 & 0.014 \\
\hline \multicolumn{5}{|l|}{ NIa-Ring 4} \\
\hline Pearson corr. & $0.4^{*}$ & 0.26 & $0.36 *$ & $0.4^{*}$ \\
\hline $\mathrm{p}$-value & 0.01 & 0.1 & 0.028 & 0.014 \\
\hline \multicolumn{5}{|l|}{ N2a-Ring 2} \\
\hline Pearson corr. & $0.31 *$ & $0.39 *$ & $0.34 *$ & $0.32 *$ \\
\hline $\mathrm{p}$-value & 0.06 & 0.016 & 0.037 & 0.04 \\
\hline \multicolumn{5}{|l|}{ N2a-Ring 3} \\
\hline Pearson corr. & $0.4^{*}$ & $0.4^{*}$ & $0.39 *$ & $0.39 *$ \\
\hline $\mathrm{p}$-value & 0.013 & 0.013 & 0.016 & 0.017 \\
\hline \multicolumn{5}{|l|}{ N2a-Ring 4} \\
\hline Pearson corr. & $0.35^{*}$ & $0.35^{*}$ & $0.37 *$ & $0.39 *$ \\
\hline $\mathrm{p}$-value & 0.035 & 0.032 & 0.021 & 0.017 \\
\hline
\end{tabular}

$\mathrm{CT}$ : the mean macular choroidal thickness; NIa: the mean amplitudes of NI wave at 6-month; N2a: the mean amplitudes of N2 wave at 6 -month; Pearson corr.: Pearson correlation coefficient; ${ }^{*}$ Correlation coefficient values $\geq \pm 0.3$.

In correlation analysis between CT and amplitudes of N2 waves for each ring, significant positive correlations between $\mathrm{CTs}$ and the mean amplitudes of the 2nd, 3rd and 4th ring N2 waves at 6-month were seen yet again only in the healthy group (Table 6).

Significant correlations were not observed between CT and other mf-ERG parameters for both groups.

\section{Correlation between OCT Parameters and CT}

There were significant negative correlations between ACDR and VCDR and CTs only in the healthy group (Table 7).

Significant relationships between $\mathrm{CTs}$ and other $\mathrm{ONH}$ OCT parameters were not found for both groups in this study.

\section{Discussion}

Due to recent developments in imaging methods, in the early stages of primary open-angle glaucoma, optic nerve OCT parameters can be recorded with high resolution and precision. In addition, the thickness of the choroid structures can be imaged in high resolution with the development of EDIOCT technology (19). As mentioned in the introduction part, technological developments can provide opportunities to support the different views about the pathophysiology of glaucoma and to reveal them in more detail. One of these views is called vascular pathogenesis theory. According to this theory, it is claimed that both the outer retinal layers and lamina cribrosa have a vascular supply from the choroid either directly or through collaterals and choroidal blood flow reduction plays an important role in the pathophysiology of glaucoma (20).

Different opinions are presented in the literature on how choroidal thickness is affected in glaucoma cases (I I-I5). In many studies in the past, subfoveal, temporal and nasal CTs were measured and evaluated separately in glaucoma cases. However, in recent years, peripapillary choroidal thickness measurements have been performed. Due to these measurements, significant changes and their correlations between ocular parameters were observed in glaucoma $(2 \mathrm{I}, 22)$. Many previous reports have suggested that no association between GON and subfoveal CT exists $(23,24)$. However, it should be noted that $\mathrm{ONH}$ is the last retina point in where the ganglion cell axons are collected and leave the retina. The first affected nerve cells in the POAG are known to be the ganglion cells, and about half of these cells are located in the parafoveal region (25). In other words, it can be thought that morphological changes of the ONH of POAG are the last reflection of nerve damages in the retina occurring in the previous nerve stages due to elevated IOP. Therefore, we have designed this study not only to evaluate the parameters of the $\mathrm{ONH}$ and its circumference but also to evaluate the parameters for detecting changes in possible previous 
Table 7. The correlations between choroidal thicknesses and cup-to-disc ratios in the healthy group

\begin{tabular}{|c|c|c|c|c|}
\hline ACDR & CT baseline & CT I-month & CT 3-month & CT 6-month \\
\hline \multicolumn{5}{|l|}{ Baseline } \\
\hline Pearson corr. & $-0.31 *$ & -0.29 & $-0.34 *$ & $-0.33^{*}$ \\
\hline $\mathrm{P}$-value & 0.04 & 0.05 & 0.02 & 0.03 \\
\hline \multicolumn{5}{|l|}{ I-month } \\
\hline Pearson corr. & -0.23 & -0.24 & -0.27 & -0.27 \\
\hline $\mathrm{P}$-value & 0.15 & 0.13 & 0.08 & 0.08 \\
\hline \multicolumn{5}{|l|}{ 3-month } \\
\hline Pearson corr. & $-0.33^{*}$ & $-0.32 *$ & $-0.34 *$ & $-0.33 *$ \\
\hline $\mathrm{P}$-value & 0.03 & 0.04 & 0.02 & 0.03 \\
\hline \multicolumn{5}{|l|}{ 6-month } \\
\hline Pearson corr. & -0.28 & -0.27 & $-0.3^{*}$ & -0.29 \\
\hline $\mathrm{P}$-value & 0.06 & 0.07 & 0.04 & 0.05 \\
\hline \multicolumn{5}{|l|}{ VCDR } \\
\hline \multicolumn{5}{|l|}{ Baseline } \\
\hline Pearson corr. & $-0.31 *$ & $-0.31 *$ & $-0.34 *$ & $-0.32 *$ \\
\hline $\mathrm{P}$-value & 0.04 & 0.04 & 0.02 & 0.03 \\
\hline \multicolumn{5}{|l|}{ I-month } \\
\hline Pearson corr. & -0.21 & -0.21 & -0.25 & -0.25 \\
\hline $\mathrm{p}$-value & 0.18 & 0.17 & 0.1 & 0.1 \\
\hline \multicolumn{5}{|l|}{ 3-month } \\
\hline Pearson corr. & $-0.34 *$ & $-0.31 *$ & $-0.34 *$ & $-0.32 *$ \\
\hline $\mathrm{p}$-value & 0.02 & 0.04 & 0.04 & 0.03 \\
\hline \multicolumn{5}{|l|}{ 6-month } \\
\hline Pearson corr. & -0.28 & $-0.3^{*}$ & $-0.31 *$ & -0.29 \\
\hline $\mathrm{P}$-value & 0.05 & 0.04 & 0.04 & 0.05 \\
\hline
\end{tabular}

$A C D R=$ average cup to disc ratio; $C T$ : the mean macular choroidal thickness; $V C D R=$ vertical cup to disc ratio; Pearson corr.: Pearson correlation coefficient; $*$ Correlation coefficient values $\geq \pm 0.3$.

neuronal stages. In addition, this study was designed to evaluate macular CT over a single value with the mean of the regions rather than individual measurements concerning a general evaluation, such as average RNFL value assessment. The aim of this design was to minimize the impact of the regional changes on the overall assessment. In our study, despite the cases being early-stage POAG and the initiation of antiglaucoma treatment, the mean $\mathrm{m}-\mathrm{CTs}$ were found to be thinner than the healthy group in all periods. Moreover, it was observed that despite decreasing IOP with medication, there was a significant reduction in the mean CTs only in the POAG group during a 6-month period. In addition to the decrease in the mean CT in the POAG group, the coexistence of the decrease in BCVAs with the change of ONH OCT parameters in the POAG group may support the importance of choroidal blood supply in the glaucoma pathogenesis. Given that the mean m-CTs in the POAG group continued to decrease although a decrease in IOP with antiglaucoma treatment may denote that other factors may play a role in the relationship between CT and IOP. A debatable issue that should be mentioned in this study is whether the reduction in the mean CTs in the POAG group was a cause or a result of glaucoma.

ONH OCT parameters play an important role in the follow-up of glaucoma. In this study, significant differences were found between POAG and healthy groups as expected in all parameters except the disc area. However, the mean RNFL thicknesses decreased significantly solely in the POAG group during the study period. According to the results of this study, it can be concluded that the thickness of the retinal nerve fiber layer is important concerning follow-up as early findings. When the RNFL thicknesses were evaluated according to the quadrants, only inferior quadrant (RNFLI) thicknesses were significantly different between the two groups and RNFLI thicknesses showed a significant decrease only in the POAG group. As emphasized in the previous studies, the optic nerve fibers spreading from the inferior and superior quadrants of $\mathrm{ONH}$ to the retina are more sensitive to the increase in IOP because of their longer lengths compared to shorter nasal and temporal nerve fibers $(26,27)$. In the 
same studies, it was deduced that the thicknesses of inferior and superior RNFL quadrants were thicker. This anatomical structure may explain the decrease of RNFL thicknesses in only inferior quadrants in the early period of this study. In our study, only the inferior quadrant RNFL was found to be more sensitive to the change in intraocular pressure because of its longer spread. This result emphasizes that the evaluation of RNFLI thickness in the early stages of glaucoma may be an important parameter concerning follow-up. In a study evaluating peripapillary CT in glaucoma cases, the CT in the inferior quadrant was found to be thinner than others (2I). This result supports our conclusion that the inferior quadrant is the first affected quadrant.

It is accepted that wave components are mostly formed by photoreceptor and bipolar cells in mf-ERG, which is an electrophysiological test that can give information about the electrical activity of many retinal regions (28). In the meantime, it should be noted that the main vascular supply of the photoreceptor cells is mainly from the choroidal vessels. Therefore, cellular damage due to choroidal vascular disorders is expected to primarily appear in photoreceptor cells. This suggests that the affected mf-ERG response in the early stages of glaucoma is worthy of investigation. However, bipolar cells in the inner retinal layer also contribute to the mf-ERG wave components. Many studies on this issue report that mf-ERG can provide valuable information concerning progression, especially in the early stages of glaucoma (29, 30). These studies suggest that during the early stages of glaucoma, electrical activity may be affected before anatomical and morphological changes in the macula. However, in our study, there were no significant differences between the POAG group and the healthy group concerning the four rings. Besides, when the significant changes in the process were examined, it was observed that the absolute values of the amplitude of the first ring $\mathrm{N} 2$ and the third ring NI negative waves decreased only in the POAG group. In this study, although there were significant changes in ONH OCT parameters in the POAG group, there was no significant difference between the two groups in $\mathrm{mf}-E R G$ parameters. This indicates that the evaluation of $\mathrm{mf}$-ERG concerning early cell function should be questioned. Given that mf-ERG findings did not correlate completely with other parameters in this study can be explained by that ganglion cells, which have less contribution to electrical activity, were the first to be affected in the POAG. The correlations of mf-ERG with ONH OCT parameters should be questioned in studies reporting significant mf-ERG changes in the pre perimetry stage. Do the pre-perimetric changes in the mf-ERG parameters occur before or after the change in OCT parameters in glaucoma? For example, in our study, despite the changes in some OCT parameters and CTs, there was no overall change in mf-ERG parameters. If the cells that contribute to mf-ERG waves are considered, an overall change in $\mathrm{mf}$-ERG parameters should not be expected in the early stages of glaucoma. However, significant reductions in some parafoveal ring parameters in the POAG group necessitate more detailed research on this issue. Given that the amplitudes of N2 wave in Ring-I and the amplitudes of NI wave in Ring- 3 only decreases in the POAG group makes the possibility of some mf-ERG parameter changes occurring in long-term progressions in the POAG worth researching.

One of the results obtained in the correlation analysis of this study is that the mean $\mathrm{mCT}$ s were significantly and negatively correlated with only the mean ACDRs and VCDRs in ONH OCT parameters only in the healthy group. Besides, there were also other significant positive correlations between the mean CTs and the mean amplitudes of $\mathrm{NI}$ and $\mathrm{N} 2$ waves in ring-2, ring-3, and ring-4 found again only in the healthy group. Considering that the main vascular supply of photoreceptor cells is via the choroidal vessels, this relationship between the $\mathrm{CT}$ and the electrical potential of photoreceptor cells, which are responsible for the major part of the electrical potential in mf-ERG, can be considered significant. An interesting issue in this regard is that these significant relationships are determined only in the healthy group. Thus, it can be considered that after the formation of glaucoma, normally existing significant relationships were affected and correlations were impaired. In addition, even though all these parameters decreased in the POAG group, given that a correlation is absent between the mean CTs and RNFL, as well as RNFLI, it supports this opinion. In the POAG group, despite the reduction of IOPs and medication, continued the reduction of the mean CTs in the process suggests that there was no direct relationship between IOP and CT. Probably other pathophysiological processes continue to affect CT. It should also be known that although the most important factor known in glaucoma is increased IOP, multifactorial effects, which cannot be explained by only IOP, play a role in the pathophysiology of glaucoma.

In chronic diseases, such as glaucoma, changes in parameters may be better understood during longer follow-up. Although one of the aims of this study was to determine the parameter changes in the steps before the $\mathrm{ONH}$ findings in newly diagnosed glaucoma cases, some amount of affected $\mathrm{ONH}$ may exist in the process of glaucoma diagnosis. The absence of correlations between some $\mathrm{ONH}$ parameters and $\mathrm{mf}$-ERG parameters, which were detected in the healthy group, in the POAG group, prevents the explanation of the possible mechanisms that cause glaucoma before the onset of $\mathrm{ONH}$ findings. The reason for the unknowns in different types of glaucoma, as well as POAG, maybe the change of multifactorial mechanisms and predictable parameter rela- 
tionships occurring before the detection of some changes in the diagnosis phase. Of course, as a vicious cycle, generally accepted glaucoma diagnostic criteria that we use when forming our glaucoma group can include the participant to the group after some changes have started to occur. To clarify the pathophysiological processes related to POAG more clearly, there is a need for a study design that can examine earlier processes. Of course, procuring acceptance of such a design from the ethics committee of universities concerning the creation of study groups is difficult in today's conditions. Studies of experimental animal models seem to contribute more to researches in this regard.

In summary, this study showed decreased choroidal thickness in glaucoma cases, as well as the decrease in choroid thickness with the glaucoma process, were likely to continue with the probable effect of other factors. Although the changes in the ONH OCT parameters with POAG were beginning to occur, the absence of significant differences in the mf-ERG parameters in this study suggests that morphological and functional changes in the cells do not change in glaucoma at least at the earliest stage. These results make the role of mf-ERG in the early stages of glaucoma doubtful concerning progression follow-up. Given that correlations between OCT, mf-ERG parameters and CT are present in the healthy group but are absent in the POAG group supports this suspicion. The decrease in the mean CT in the glaucoma group, despite medical treatment, has shown that direct relation between IOP and CT is absent, but CT is affected by some mechanisms associated with glaucoma formation. Of course, animal experiments involving a large scale and long-term histopathological evaluation can provide a direct explanation of this mechanism. This study has also shown that RNFLI may be an important marker in the evaluation of early-stage glaucoma progression by showing reduction only in RNFLI thickness in all quadrants. In order to clarify all of these relationships more clearly, clinical studies with greater participation and longer duration are needed.

\section{Acknowledgements}

This study was presented at Afyon Kocatepe University (specialization in medicine thesis) in June 2015, Afyonkarahisar, Turkey; and also in the 49th National Congress of Turkish Ophthalmology Association, November 2015, Harbiye - Istanbul, Turkey.

Supported by the Scientific Research Projects Board Unit of Afyon Kocatepe University (13.TUS.08). The funding organization had no role in the design or conduct of this research.

\section{Disclosures}

Ethics Committee Approval: The Ethics Committee of University of Afyon Kocatepe provided the ethics committee approval for this study (2014/04-74).
Peer-review: Externally peer-reviewed.

Conflict of Interest: None declared.

Authorship Contributions: Involved in design and conduct of the study (BK, GFY); preparation and review of the study (BK, GFY, MD); data collection (BK, GFY, MD, SI, UUI); and statistical analysis (BK, GFY).

\section{References}

I. European Glaucoma Society Terminology and Guidelines for Glaucoma, 4th Edition- Chapter 2: Classification and terminology Supported by the EGS Foundation: Part I: Foreword; Introduction; Glossary; Chapter 2 Classification and Terminology. $\mathrm{Br}$ J Ophthalmol 2017;101:73-127. [CrossRef]

2. Tham YC, Li X, Wong TY, Quigley HA, Aung T, Cheng CY. Global prevalence of glaucoma and projections of glaucoma burden through 2040: a systematic review and meta-analysis. Ophthalmology 2014;121:2081-90. [CrossRef]

3. The Advanced Glaucoma Intervention Study (AGIS): 7 The relationship between control of intraocular pressure and visual field deterioration. The AGIS Investigators. The Advanced Glaucoma Intervention Study (AGIS): 7 The relationship between control of intraocular pressure and visual field deterioration. The AGIS Investigators. Am J Ophthalmol 2000;130:42940. [CrossRef]

4. Matlach J, Bender S, König J, Binder, Pfeiffer N, Hoffmann EM. Investigation of intraocular pressure fluctuation as a risk factor of glaucoma progression. Clin Ophthalmol 2018;13:9-16. [CrossRef]

5. Yarmohammadi A, Zangwill LM, Diniz-Filho A, Suh $M H$, Manalastas PI, Fatehee N, et al. Optical coherence tomography angiography vessel density in healthy, glaucoma suspect and glaucoma eyes. Invest Ophthalmol Vis Sci 2016;57:OCT45I-9.

6. Lévêque PM, Zéboulon P, Brasnu E, Baudouin C, Labbé $A$, et al. Optic disc vascularization in glaucoma: value of spectral-domain optical coherence tomography angiography. J Ophthalmol 2016;20।6:69567I7. [CrossRef]

7. Yu PK, Cringle SJ, Yu DY. Correlation between the radial peripapillary capillaries and the retinal nerve fibre layer in the normal human retina. Exp Eye Res 2014;129:83-92. [CrossRef]

8. Mansoori T, Gamalapati J, Sivaswamy J, Balakrishna N Optical coherence tomography angiography measured capillary density in the normal and glaucoma eyes. Saudi J Ophthalmol 2018;32:295-302. [CrossRef]

9. Mase T, Ishibazawa A, Nagaoka T, Yokota H, Yoshida A. Radial peripapillary capillary network visualized using wide-field montage optical coherence tomography angiography. Invest Ophthalmol Vis Sci 2016;57:OCT504-10. [CrossRef]

10. Kiel JW. The Ocular Circulation. Texas: Morgan \& Claypool Life Sciences; 2010.

I I. Park JH, Yoo C, Kim YY. Peripapillary choroidal thickness in untreated normal-tension glaucoma eyes with a single-hemifield retinal nerve fiber layer defect. Medicine (Baltimore) 
2018;97:el I00I. [CrossRef]

12. Kiyota N, Kunikata H, Shiga Y, Omodaka K, Nakazawa T. Relationship between laser speckle flowgraphy and optical coherence tomography angiography measurements of ocular microcirculation. Graefes Arch Clin Exp Ophthalmol 2017;255:1633-42. [CrossRef]

13. Lee EJ, Kim S, Hwang S, Han JC, Kee C. Microvascular compromise develops following nerve fiber layer damage in normaltension glaucoma without choroidal vasculature involvement. J Glaucoma 2017;26:216-22. [CrossRef]

14. Wang W, Zhang $X$. Choroidal thickness and primary open-angle glaucoma: a cross-sectional study and meta-analysis. Invest Ophthalmol Vis Sci 2014;55:6007-14. [CrossRef]

15. Wang YX, Xu L, Shao L, Zhang YQ, Yang H, Da Wang J, et al. Subfoveal choroidal thickness and glaucoma. The Beijing Eye Study 20I I. PLoS One 2014;9:el0732I. [CrossRef]

16. Robson AG, Nilsson J, Li S, Jalali S, Fulton AB, Tormene AP, et al. ISCEV guide to visual electrodiagnostic procedures. Doc Ophthalmol 2018;136:I-26. [CrossRef]

17. Brandão LM, Monhart M, Schötzau A, Ledolter AA, PalmowskiWolfe AM. Applying a New Automed Perimetry Pattern Based on the Stimulus Distribution of the Multifocal ERG to Improve Structure-Function Investigation in Glaucoma. J Ophthalmol 2017;2017:8780934. [CrossRef]

18. Gao M, Wang Y, Liu W, Liu L, Yan W, Liu J, et al. Assessment of macular function in patients with idiopathic Epiretinal membrane by multifocal Electroretinography: correlation with visual acuity and optical coherence tomography. BMC Ophthalmol 2017; 17:221. [CrossRef]

19. Spaide RF, Koizumi H, Pozzoni MC. Enhanced depth imaging spectral-domain optical coherence tomography. Am J Ophthalmol 2008; | 46:496-500. [CrossRef]

20. Weinreb RN, Aung T, Medeiros FA. The pathophysiology and treatment of glaucoma: a review. JAMA 2014;3 II:1901-II.

2I. Pablo LE, Cameo B, Bambo MP, Polo V, Larrosa JM, Fuertes MI, et al. Peripapillary Choroidal Thickness Analysis Using SweptSource Optical Coherence Tomography in Glaucoma Patients: A Broader Approach. Ophthalmic Res 2018;59:7-13. [CrossRef]

22. Lee SH, Lee EJ, Kim TW. Topographic correlation between juxtapapillary choroidal thickness and parapapillary deeplayer microvasculature dropout in primary open-angle glaucoma. $\mathrm{Br} J$ Ophthalmol 2018;102:1 134-40.

23. Banitt M. The choroid in glaucoma. Curr Opin Ophthalmol 2013;24:125-9. [CrossRef]

24. Abegão Pinto L, Willekens K, Van Keer K, Shibesh A, Molenberghs G, Vandewalle E, et al. Ocular blood flow in glaucoma the Leuven Eye Study. Acta Ophthalmol 2016;94:592-8. [CrossRef]

25. Quigley HA, Dunkelberger GR, Green WR. Retinal gangli- on cell atrophy correlated with automated perimetry in human eyes with glaucoma. Am J Ophthalmol 1989; 107:453-64. [CrossRef]

26. Pomorska M, Krzyżanowska-Berkowska P, Misiuk-Hojło M, Zając-Pytrus H, Grzybowski A. Application of optical coherence tomography in glaucoma suspected eyes. Clin Exp Optom 2012;95:78-88. [CrossRef]

27. Caprioli J, Nouri-Mahdavi K, Law SK, Badalà F. Optic disc imaging in perimetrically normal eyes of glaucoma patients with unilateral field loss. Trans Am Ophthalmol Soc 2006; 104:202-I I.

28. Kuhn F, Witherspoon CD, Skalka H, Morris R. Improvement of siderotic ERG. Eur J Ophthalmol 1992;2:44-5. [CrossRef]

29. Wilsey LJ, Fortune B. Electroretinography in glaucoma di- agnosis. Curr Opin Ophthalmol 2016;27:I I8-24. [CrossRef]

30. Brandao LM, Ledolter AA, Monhart M, Schötzau A, PalmowskiWolfe AM. Ganglion cell layer segmentation and the two-flash multifocal electroretinogram improve structure function analysis in early glaucoma. Graefes Arch Clin Exp Ophthalmol 2017;255:199|-2000. [CrossRef] 